\title{
APPLICATION OF A BALANCED ADDER-SUBTRACTOR IN THE SOLUTION OF SIMULTANEOUS LINEAR EQUATIONS
}

\author{
M. D. Tyona, O. J. Tsor and R. L. Njinga \\ Department of Physics, Faculty of Science, Benue State University, Makurdi, Nigeria.
}

(Submitted: 30 November, 2006; Accepted: 3 June, 2007)

\section{Abstract}

A balanced adder-subtractor employing operational amplifier is used to solve simultaneous linear equations. A pair of simultaneous linear equation with two unknowns have been considered for the design of the Galanced adder-subtractor operated from a 6-volts d.c power source. A general purpose operational amplifier, $\mu$ A 741 of the 8- Cead mini DIP type is chosen for the design. It's low power requirement, high gain, wide range of operating voltage, absence of "Catch-up" tendencies and high common mode voltage range are some of the prime advantages of the $\mu A 741$ considered for it's choice in this work. The performance of the designed circuit has been evaluated for input voltages, $20 \mathrm{~V}$ and $5 \mathrm{~V}$ respectively. The output obtained from the system, $x_{0}=0.623 \mathrm{~V}$ and $y_{0}=0.754 \mathrm{~V}$ on the scale of $0.1 \mathrm{~V}=1$ confirmed the solution of the pair of simultaneous equation.

Keywords : Balanced adder-subtractor, linear equations and operational amplifier

\section{Introduction}

Operational amplifiers are widely used in electronic and measurement systems. They are among the active components of electronic circuits. The application of operational amplifier in electronic circuits have received much attention due to their potential advantages in terms of miniaturization, ease of design and high frequency performance (Shinde, 2002).

They can be configured to execute most mathematical operations such as integration, differentiation and simultaneous linear and quadratic equations among others.

A balanced adder-subtractor is an electronic circuit that have employed the use of operational amplifier to solve simultaneous linear equations and some other related operations. As illustrated in fig. 1, the balanced adder-subtractor circuit is a generalization of the differential input amplifier. Its operation is based on voltage Summation and subtraction. In it's best operating conditions, the inverting and noninverting gains must be balanced; and hence the name.
A linear equation in two variables of the form $a x+b y=c$ has no unique solution. This is an equation of a straight line in which several points with co-ordinates $(x, y)$ on the line will satisfy the equation (Clayton, 1987). However, for a pair of simultaneous equations, a unique solution can be obtained so long as the equations are not those of parallel lines.

Several methods of solving such equations include the graphical, elimination and substitution methods. In most cases, the solution can be easily obtained by simple mathematical manipulations of multiplication, addition and subtraction. In some cases, they are not very simple to solve. Circuits for solving simultaneous linear equations have been published (Asaolu, 1995). These circuits used operational amplifier as an inverting amplifier and balance was not achieved. Also the circuits were configured for specific cases.

The objective of this work is to design and construct a balanced adder-subtractor, an analog circuit which will help in solving pairs of simultaneous linear equations. A pair 
of linear equation $2 x+3 y=40$ and $2 x+3 y=5$ are to be considered for this design. However, the circuit will be modified for any pair of linear equations.
A generalized balanced adder-subtactor circuit employing an operational amplifier, $\mu A 741$ as the active component is shown in Fig 1.

\section{Technical Design of the Balanced Adder-Subtractor Circuit}

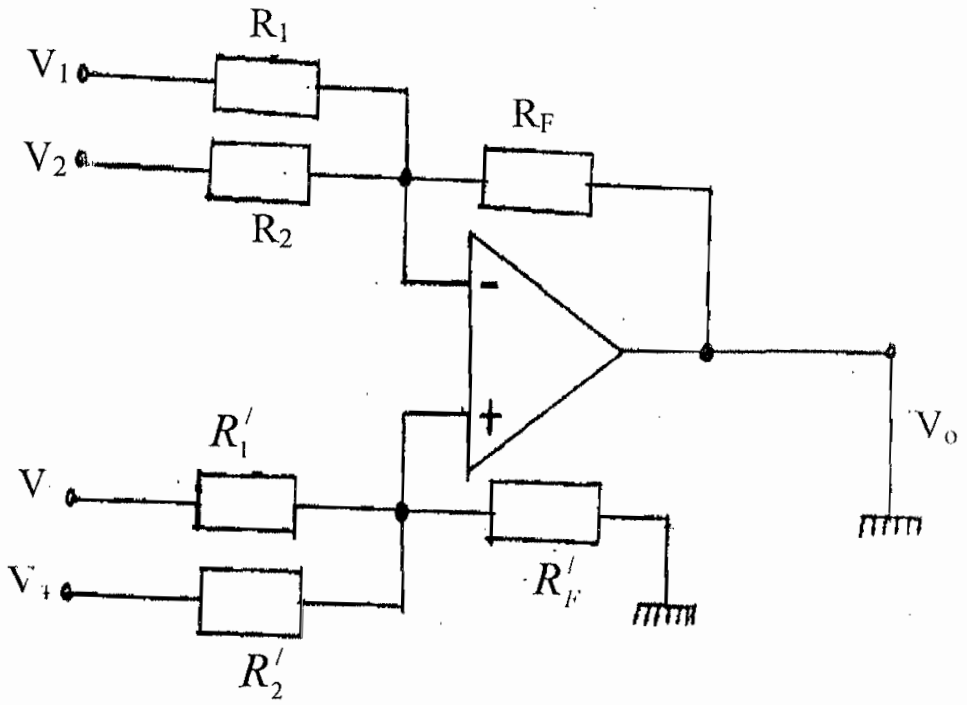

Fig 1: A generalized balanced adder-subtractor circuit

For a balanced adder-subtractor, the loop gains for inverting and non-inverting are equal; that is (F'ig. 1)

$$
\frac{R_{f}}{R_{1}}+\frac{R_{f}}{R_{2}}+\cdots+\frac{R_{f}}{R_{m}}=\frac{R_{f}^{\prime \prime}}{R_{1}^{\prime}}+\frac{R_{f}^{\prime}}{R_{2}^{\prime}}+\cdots+\frac{R_{f}^{\prime}}{R_{n}^{\prime}}
$$

The output voltage $V_{0}$ for both inverting and non-inverting inputs is given as

$$
V_{o}=\left(V_{3} \frac{R_{f}^{\prime}}{R_{1}^{\prime}}+V_{4} \frac{R_{f}^{\prime}}{R_{2}^{\prime}}\right)-\left(V_{1} \frac{R_{f}}{R_{1}}+V_{2} \frac{R_{f}}{R_{2}}\right)
$$

The pair of simultaneous linear equation

$$
\begin{aligned}
& 2 x+3 y=40 \\
& 2 x+y=5
\end{aligned}
$$

can be expressed as

$$
\begin{aligned}
& x=20-1.5 y \\
& y=5-2 x
\end{aligned}
$$

The mathematical operations involved in the equation for $x$ and $y$ are those of addition and multiplication by constant coefficients which can easily be performed by the use of electrical signals.

In analog computations, the numbers representing the variables in a problem are represented continuously by voltages. We decide on a scale of $0.1 \mathrm{~V}=1$ that will contain all of our possible solutions over a given range of coefficients which are to be used. Thus, a number $x=15$ would correspond to $1.5 \mathrm{~V}$ on the $\mathrm{x}$-output.

A comparison of equations (2) and (5) shows that $x=V_{0}, V_{3}=20 \mathrm{~V}, V_{1}=y$

Hence, equation (5) is expressed as

$$
x=20 \frac{R_{f}^{\prime}}{R_{1}^{\prime}}-\frac{R_{f}}{R_{1}} y^{\prime}
$$

The positive number will be fed in on the noninverting input with a gain $R_{f}^{\prime} / R_{1}^{\prime}=1$. For the gain of one, we set $R_{f}^{\prime}=R_{1}^{\prime}$, and also for convenience, $R_{f}=R_{f}^{\prime}$. Hence, $R_{f}=R_{f}^{\prime}=R_{1}^{\prime}=100 \mathrm{k} \Omega$ and 
$\mathrm{R}_{1}=68 \mathrm{k} \Omega$.

We now connect the first stage of the amplifier as shown in fig 2. $R_{x}$ is added to the non-inverting input such that $R_{f}^{\prime} / R_{x}^{\prime}=5$. Thus, the irverting and non-inverting gains each equal to 1.5 ; that is $R_{x}=200 \mathrm{k} \Omega$.

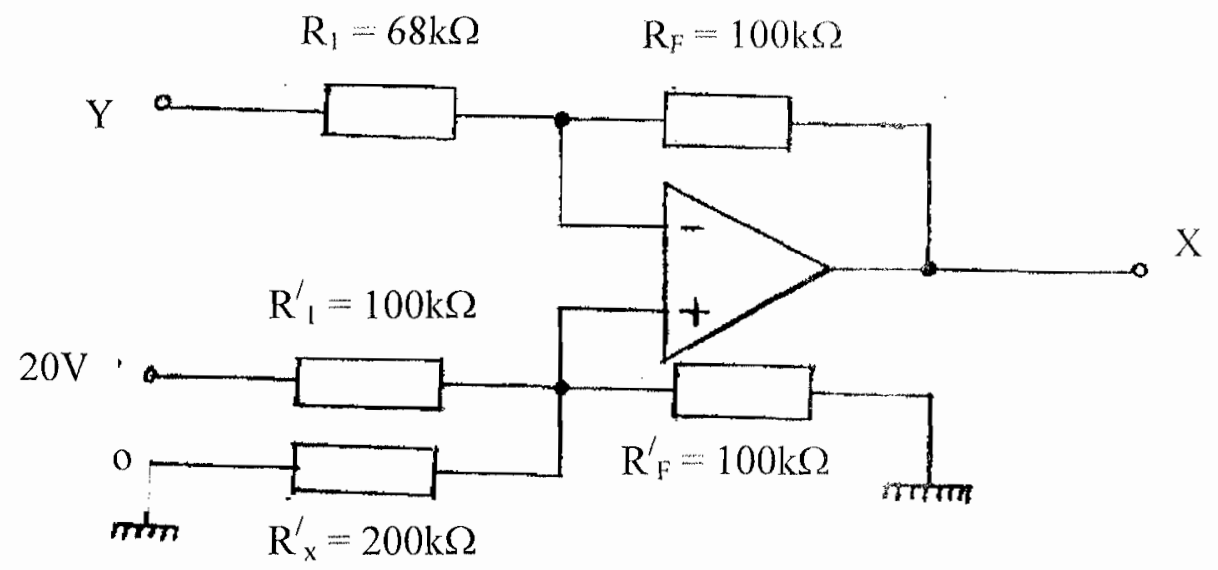

Fig 2: $x=20-1.5 y$ connection.

A second stage of amplifier is set to solve for $y$. Since $y=5-2 x$, it follows that

$$
y=5 \frac{R_{f}^{\prime}}{R_{1}^{\prime}}-\frac{R_{f}}{R_{1}} x
$$

Where $\mathrm{R}_{\mathrm{f}} / \mathrm{R}_{1}=2$ and $R_{f}^{\prime} / R_{1}^{\prime}=1$. Selecting $R_{f}=R_{f}{ }^{\prime}=100 \mathrm{k} \Omega$, hence $R_{1} \approx 47 \mathrm{k} \Omega$ and
$R_{1}^{\prime}=100 \mathrm{k} \Omega$. This gives an inverting gain of two, which is not equal to the non-inverting gain of one. To achieve balance, a zero input with a gain of one is added to the noninverting side, that is $R_{f}^{\prime} / R_{y}^{\prime}=1$, hence $R_{y}^{\prime}=100 \mathrm{k} \Omega$. Thus the second stage of the amplifier is connected as shown in fig 3 .

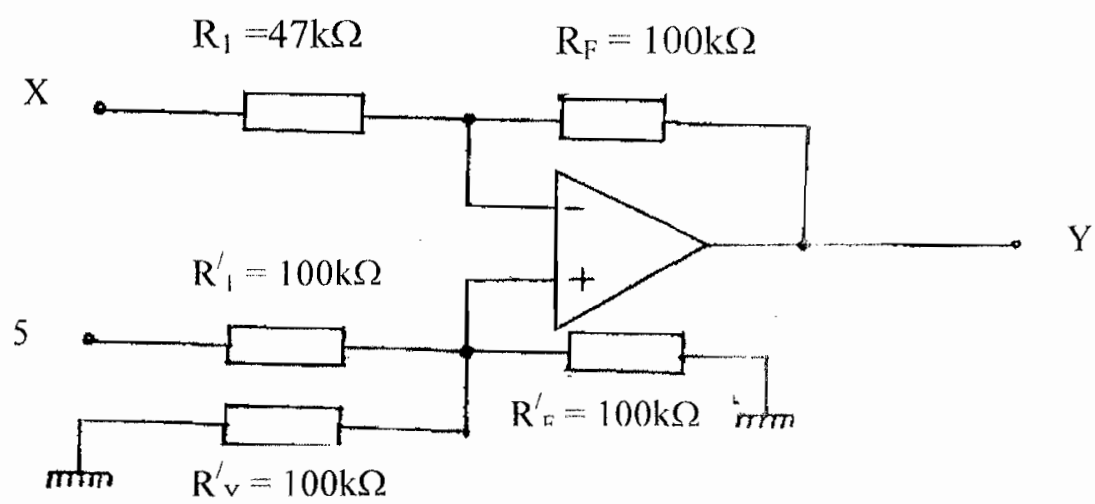

Fig 3: $y=5-2 x$ Connection. 
For any pair of simultaneous linear equation, the unique solutions of $x$ and $y$ are interdependent. Hence, the two stages of the amplifier are cascaded back to back such that the output of the amplifier solving for $x$ is connected to the $x$-input of the amplifier solving for $y$ while the output of the amplifier solving for $y$ is a $y$-input for the amplifier solving for $\mathrm{x}$. This is shown in fig 4 .

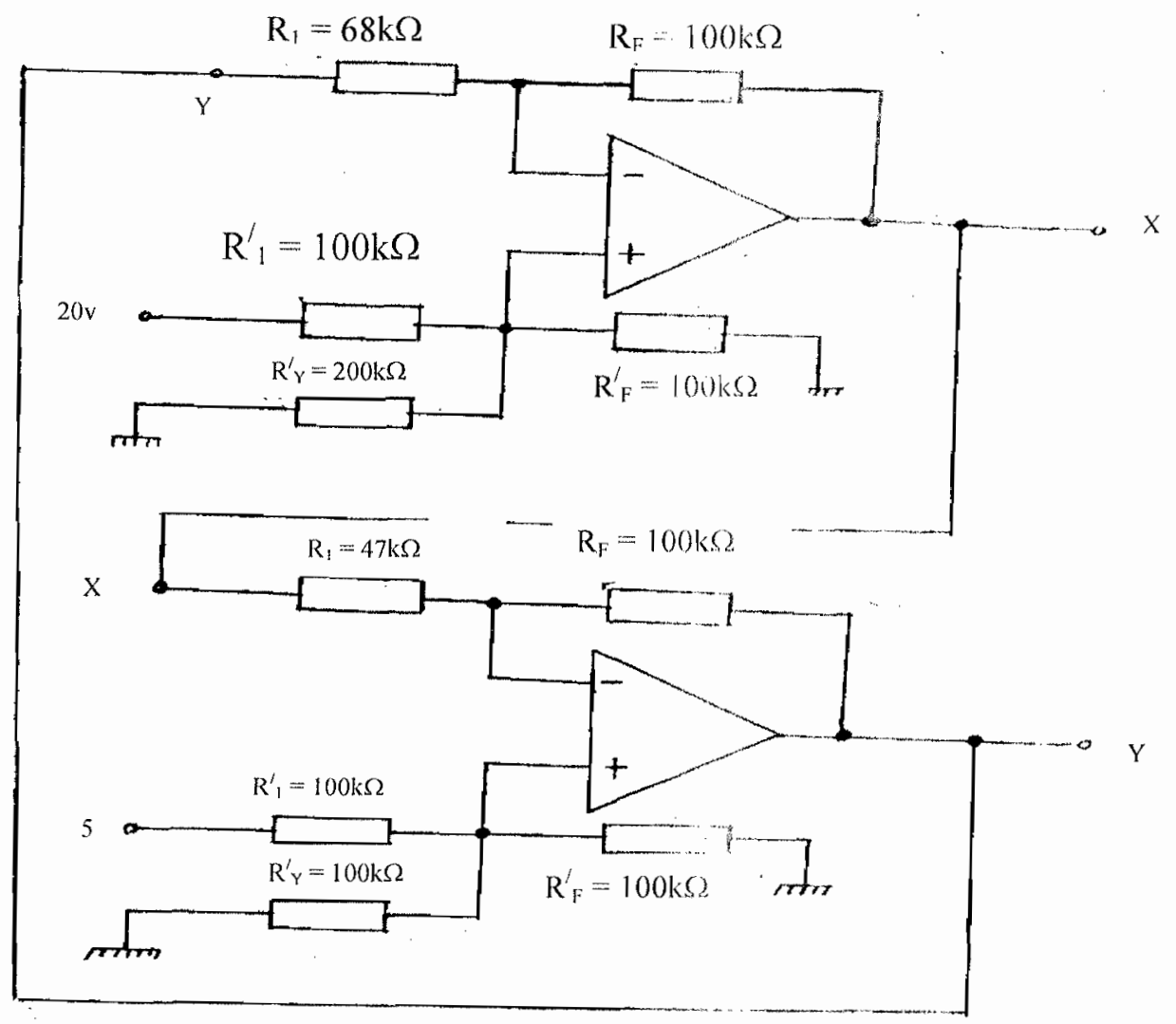

Fig 4: Circuit for solving simultaneous equation $2 x+3 y=40$ and $2 x+y=5$.

The electronic components were coupled on a single circuit board as one circuit of the designed Adder-Subtractor.

\section{Results and Discussion}

Results obtained for the given pair of simultaneous equations are stated in Table one.

Table 1: Results obtained the output of Fig 4 and the algebraic computation

\begin{tabular}{|l|l|l|l|}
\hline$x_{0}$ & $y_{0}$ & $x_{a}$ & $y_{a}$ \\
\hline 0.623 & 0.754 & 6.25 & 7.50 \\
\hline
\end{tabular}

$x_{0}, y_{0}$ are the results obtained from the output of the circuit for $x$ and $y$ respectively, while $x_{a}$ and $y_{a}$ are the corresponding results obtained from algebraic computations. The two results compare favorably well. The slight differences may be due to experimental errors in the reading of oscilloscope.

The circuit has been modified to solve any given pair of simultaneous equation as shown in fig (5). Potentiometers with wide range of resistances are used as the feedback networks so that they can be varied continuously to provide the required gains at various input signals. Also $R_{x}{ }^{1}$ and $R_{y}{ }^{1}$ are been substituted with variable resistors so that balance can be obtained at any gain. 


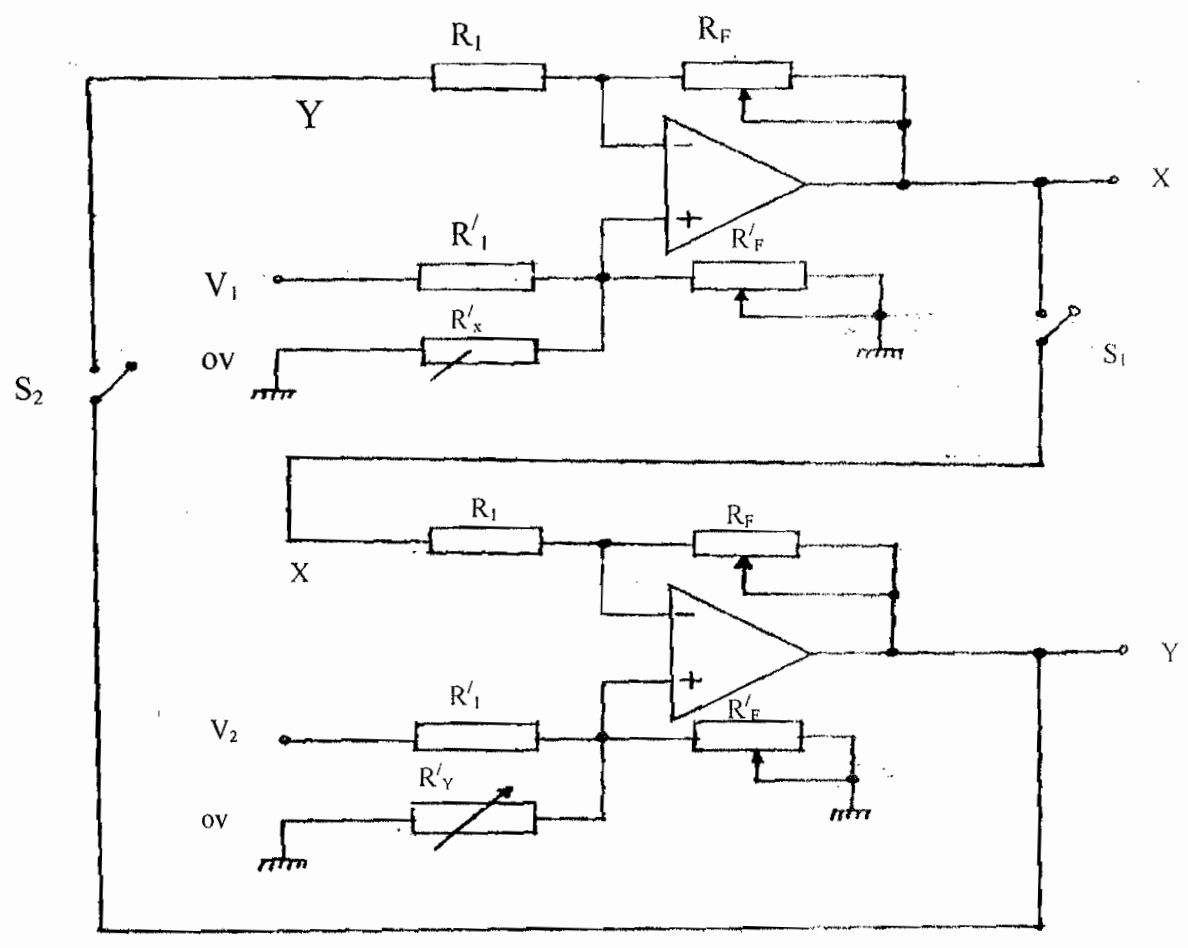

Fig 5: Modified circuit to solve any pair of simultaneous equation.

\section{References}

Clayton, G.B; (1987): Operational Amplifiers; Butterworth, London, Pp 252-261.

Faulkenberry, L.M; (1977): An introduction to operational Amplifiers; John Wiley and Sons, New York.

Grinich, V.H and H.J. Jackson, (1975): Introduction to Integrated Circuits, McGraw-Hill Books Coy; New York.

Holt, C.A; (1978): Electronic circuits (Digital and Analogue); John Wiley and Sons, Canada.
Horowitz, P and W. Hill (1995): The Art of Electronics, Cambridge Press, Cambridge.

Sentura, S.C and B.D. Wedlock (1975): Electronic Circuits and Application, Wiley International, London,

Shinde, G.N, Kadam, A.B, Karumbhatte, S.B, Patil, P.B(2002): The Study of Active- $R$ Second Order Filter Using Feedback At Non-inverting Terminal,Bulletin of Pure and Applied Sciences Vol. 21D (No. 1), P 23-31. 